\title{
On certain coefficients of Drinfeld-Goss eigenforms with power eigenvalues
}

\author{
Ahmad El-Guindy ${ }^{1,2}$ (D) $^{*}$ and Aleksandar Petrov ${ }^{3}$
}

\author{
*Correspondence: \\ a.elguindy@gmail.com \\ 1 Department of Mathematics, \\ Faculty of Science Cairo University, \\ Giza 12613, Egypt \\ 2 Present Address: Texas A\&M \\ University at Qatar, Science \\ Program, Doha 23874, Qatar \\ Full list of author information is \\ available at the end of the article
}

\begin{abstract}
We show that a certain family of the coefficients of a Drinfeld-Goss modular form with certain power eigenvalues for the Hecke operators at degree 1 primes the can be expressed as polynomial multiples of the first possible non-zero coefficient of that form. Along the way, we obtain some interesting combinatorial properties regarding difference operators in finite characteristic.
\end{abstract}

Keywords: Drinfeld-Goss modular forms, Hecke operators, Eigenform coefficients, Difference operators

2010 Mathematics Subject Classification: 11F52, 11F25

\section{Introduction}

Let $p$ be a prime number. Let $q:=p^{r}$, with $r$ a positive integer, and consider the finite field $\mathbb{F}_{q}$. Let $A:=\mathbb{F}_{q}[\theta], K:=\mathbb{F}_{q}(\theta)$. We write $A_{+}$for the set of monic polynomials in $A$.

For $a \in A$, we define $|a|:=q^{\operatorname{deg} a}$ and extend this absolute value to $K$. The completion of $K$ with respect to this absolute value, denoted by $K_{\infty}$, is given by $K_{\infty}=K\left(\left(\theta^{-1}\right)\right)$. Let $\mathbb{C}_{\infty}$ be the completion of a fixed algebraic closure of $K_{\infty}$. The field $\mathbb{C}_{\infty}$ is complete and algebraically closed. The rigid analytic space $\mathbb{C}_{\infty} \backslash K_{\infty}$ is the function field analogue of the complex upper half-plane, and we shall refer to it as the the Drinfeld upper half-plane.

Let $M_{k, m}$ denote the set of Drinfeld-Goss modular forms of weight $k$, type $m$, for $\mathbf{G L}_{2}(A)$ over $\mathbb{C}_{\infty}$ (see [3] or [2] §5). The type $m$ is a residue class modulo $q-1$, and typically $m$ is chosen to be the canonical representative $0 \leq m<q-1$. However, for this paper it will be convenient to occasionally choose $2 \leq m \leq q$ (thus types 0 and 1 become viewed as types $q-1$ and $q$, respectively). We shall clarify which convention is being used whenever needed.

Next, we set

$$
u(z):=\frac{1}{\tilde{\pi}} \sum_{a \in A} \frac{1}{z+a},
$$

where $\tilde{\pi}$ is a fixed choice of a fundamental period of the Carlitz module. Note that $u=u(z)$ is the usual uniformizer at the 'infinite' cusp of the Drinfeld upper half-plane. If $f$ is an element of $M_{k, m}$, and $z \in \mathbb{C}_{\infty}$ is an element for which $|z|_{i}:=\inf _{a \in K_{\infty}}|z-a|$

(C) 2016 El-Guindy and Petrov. Open Access This article is distributed under the terms of the Creative Commons Attribution 4.0 International License (http://creativecommons.org/licenses/by/4.0/), which permits unrestricted use, distribution, and reproduction in any medium, provided you give appropriate credit to the original author(s) and the source, provide a link to the Creative Commons license, and indicate if changes were made. 
is sufficiently large $\left(|z|_{i}\right.$ is a function field analogue of the 'imaginary distance'), then we have

$$
f(z)=\sum_{n=0}^{\infty} a_{n} u(z)^{n}, \quad a_{n} \in \mathbb{C}_{\infty},
$$

and this expansion determines $f$ uniquely. This expansion is called the $u$-expansion of $f$ at 'infinity'. Such expansions, as well as the foundations of the theory of modular form in the Drinfeld module setting, were introduced by Goss in the seventies (see [4] and [3] for instance). If $f \in M_{k, m}$ and $a_{0}=0$, then $f$ is called cuspidal, and if both $a_{0}=0$ and $a_{1}=0$ we call $f$ double-cuspidal. We shall denote the subspace of cuspidal forms inside $M_{k, m}$ by $S_{k, m}$, and the space of double-cuspidal forms by $M_{k, m}^{2}$.

Let $u_{a}:=u(a z)$. The function $u_{a}$, for $a \in A_{+}$, can be expanded into a $u$-series with coefficients in $A$ (see [2] (7.3)). Following standard notation we set

$$
g:=1-\left(\theta^{q}-\theta\right) \sum_{a \in A_{+}} u_{a}^{q-1} \in M_{q-1,0}, \quad h:=\sum_{a \in A_{+}} a^{q} u_{a} \in S_{q+1,1} .
$$

The forms $g$ and $h$ generate the algebra of modular forms of all weights and types.

Let $\mathfrak{p}$ be a prime ideal of $A$. By a slight abuse of notation, we will also denote by $\mathfrak{p}$ the (unique) monic irreducible generator of the ideal $\mathfrak{p}$. Let $f \in M_{k, m}$ and let $\mathcal{T}_{\mathfrak{p}, k}$ denote the

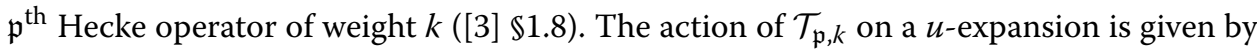
(see [2] (7.3)):

$$
\mathcal{T}_{\mathfrak{p}, k}\left(\sum_{n=0}^{\infty} a_{n} u^{n}\right)=\mathfrak{p}^{k} \sum_{n=0}^{\infty} a_{n} u_{\mathfrak{p}}^{n}+\sum_{n=0}^{\infty} a_{n} G_{n, \mathfrak{p}}(\mathfrak{p} u),
$$

where $G_{n, \mathfrak{p}}(X)$ is the $n$-th Goss polynomial of the finite lattice formed by the $\mathfrak{p}$-torsion of the Carlitz module (see ([2] (3.4)) for the definition of Goss polynomials of a lattice). As the form $f$ determines the weight $k$, we will usually write $\mathcal{T}_{\mathfrak{p}}$ instead of $\mathcal{T}_{\mathfrak{p}, k}$. As in the classical case, both $M_{k, m}$ and $S_{k, m}$ are stable under the Hecke action. However, in contrast with the classical case, Goss ([4] §3) observed that the space $M_{k, m}^{2}$ of double-cuspidal forms is also preserved by all $\mathcal{T}_{\mathfrak{p}}$.

A Drinfeld-Goss modular form $f \in M_{k, m}$ is called an eigenform if it is an eigenvector for all $\left\{\mathcal{T}_{\mathfrak{p}}: \mathfrak{p}\right.$ monic irreducible $\}$, i.e., $\mathcal{T}_{\mathfrak{p}} f=\lambda_{\mathfrak{p}} f$ for all $\mathfrak{p}$, with eigenvalues $\lambda_{\mathfrak{p}} \in \mathbb{C}_{\infty}$. The eigensystem of an eigenform $f$ is the collection of eigenvalues $\left\{\lambda_{\mathfrak{p}}\right\}$. For example, $g$ is an eigenform with eigensystem $\left\{\lambda_{\mathfrak{p}}=\mathfrak{p}^{q-1}\right\}$ and $h$ is an eigenform with eigensystem $\left\{\lambda_{\mathfrak{p}}=\mathfrak{p}\right\}([2] \S 7)$.

In the case of classical modular forms, it is well known that the eigenvalues completely determine the coefficients at square-free indices of a normalized Hecke cusp form $f(z)=\sum_{n=1}^{\infty} a_{n}(f) e^{2 n \pi i z}$ in a rather simple way; namely the eigenvalue at a prime $p$ appears as the $p^{\text {th }}$ Fourier coefficient $a_{p}(f)$ of that eigenform. Furthermore, if $n=\prod_{i=1}^{l} p_{i}$ is a product of distinct prime, then $a_{n}(f)=\prod_{i=1}^{l} a_{p_{i}}(f)$. These phenomena don't exactly carry over in the present setting, and there are in fact infinitely many examples of Drinfeld-Goss eigenforms of different weights which all have the same eigensystem, notably of the form $\left\{\lambda_{\mathfrak{p}}=\mathfrak{p}^{n}\right\}$, which we shall designate as power eigensystems. Nonetheless, our main result below gives (under the assumption that $q$ is prime) a precise description of an infinite family of coefficients which are determined by certain eigenvalues in a way that is independent of the weight (cf. Theorem 2.3). This extends the 
level 1 case of an analogous result of Armana [1] for forms of types 0 and 1 on $\Gamma_{0}(\mathfrak{n})$ for $\mathfrak{n} \in A$, although the proof and the nature of the resulting formulas are rather different.

We shall recall the results of Armana and state our own in Section 2. In Section 3, we study the combinatorial properties of certain natural successive difference operators on spaces of functions over $\mathbb{F}_{q}$ whose properties play an important role in deriving our results. In Section 4, we obtain a useful result on the vanishing of certain binomial coefficients appearing in the Hecke action formula for degree 1. We then combine all those ingredients in Section 5 to prove Theorem 2.3.

It is the authors' pleasure to express their gratitude to David Goss for his constant support and encouragement, and to David Goss, Rudy Perkins, and the anonymous referee for many useful comments on earlier versions of this manuscript. They are also grateful to Gebhard Böckle, Cécile Armana and Ambrus Pal for several useful discussions.

\section{Coefficients of Eigenforms}

For the remainder of the paper, we shall always assume that $q$ is a prime. We start by recalling a theorem of Armana which provides an expression for certain coefficients of doubly cuspidal Hecke eigenforms of type 0 or 1 .

Theorem 2.1. ([1] Theorem 1.2) Assume $q$ is prime, and let $\mathscr{S}$ be the set of natural integers of the form $c /(q-1)$, where $c$ is a positive integer whose sum of base $q$ digits is $(q-1)$. If $f=\sum b_{i}(f) u^{1+i(q-1)}$ is a type 1 double-cuspidal Hecke eigenform on $\Gamma_{0}(\mathfrak{n})$ for some $\mathfrak{n} \in A$, then for every $n \in \mathscr{S}$ there exists an element $s_{n}$ in the Hecke algebra on $M_{k, 1}^{2}(\mathfrak{n})$ such that

$$
b_{n}(f)=b_{1}\left(s_{n} f\right) .
$$

Furthermore, an analogue of (3) is also true for a type 0 double-cuspidal eigenform $f=\sum b_{i}(f) u^{i(q-1)}$ at coefficients $b_{n}$ for which $n \in \mathscr{S}_{0}:=\{n \in \mathscr{S}: q \nmid n\}$.

Furthermore, an explicit version of that theorem is provided in ([1] Theorem 7.2) where formulas for the Hecke algebra elements $s_{n}$ are presented in terms of sums involving operators $\mathcal{T}_{\mathfrak{p}}$ as $\mathfrak{p}$ varies over monic irreducible primes of degree at least the length of the base $q$ expansion of $c$.

Remark 2.2. While the restriction on $\mathscr{S}_{0}$ isn't present in [1], we have verified with Armana that it is indeed needed in the type 0 case. However, that omission has only minor and easily deducible consequences on the remainder of that paper. For example, the bound on the weight in ([1] Theorem 7.7(1)) should be changed to $k<q\left(q^{2}-1\right)$.

In the following theorem, we present a result in the same vein of Theorem 2.1 which applies to eigenforms in $M_{k, m}^{2}$ for any type $m$ and any weight $k$. Furthermore, we obtain that the coefficients in question are in fact all polynomial multiples of the initial one. We also note that our statements and proofs rely only on Hecke operators at degree 1 primes.

Theorem 2.3. Let $f=\sum_{i \geq 2} a_{i} u^{i}$ be a type $m$ (with $2 \leq m \leq q$ ) double-cuspidal form satisfying $\mathcal{T}_{\theta} f=\theta^{N} f$, where $N$ is a positive integer of the form $N=1+q^{N_{1}}+\cdots+q^{N_{l}}$, with 
$1 \leq l \leq q-1$. Then for each length $l$ multiset (i.e., elements may repeat) of non-negative integers $v=\left\{v_{1}, \ldots, v_{l}\right\}$, there exist polynomials $A(v ; \theta) \in \mathbb{F}_{q}[\theta]$ such that

$$
a_{1+q^{\nu_{1}}+\cdots+q^{\nu_{l}}}=A(v ; \theta) a_{1+l} .
$$

These polynomials depend only on $N$ and $v$, and they satisfy $A(v ; \theta+c)=A(v ; \theta)$ for all $c \in \mathbb{F}_{q}$, and hence $A(v ; \theta) \in \mathbb{F}_{q}\left[\theta^{q}-\theta\right]$.

Remark 2.4. It is well known that for $f=\sum a_{i} u^{i} \in M_{k, m}$, we have $a_{i}=0$ unless $i \equiv m(\bmod q-1)$. Since $1+q^{\nu_{1}}+\cdots+q^{\nu_{l}} \equiv l+1(\bmod q-1)$, a necessary condition for not all $A(v ; \theta)$ to vanish is to have $l+1=m$ and at least one multiset $\left\{v_{1}, \ldots, v_{l}\right\}$

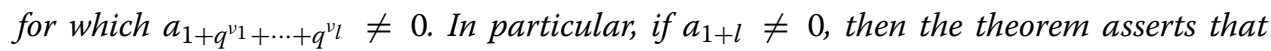
certain coefficients of the normalized form $f / a_{l+1}$ are given as polynomials depending on the eigenvalues, which can be viewed a function field analogue to how the p-th coefficient of a normalized eigenform is itself the eigenvalue of the p-th Hecke operator in the classical case. Note that the choice $2 \leq m \leq q$ conveniently allows us to apply the condition $l+1=$ $m$ uniformly to all types.

Example 2.5. When $q=3$, the form $f=h^{2} g^{2}$ belongs to the one-dimensional space of weight 12 and type 0 (equivalently, type 2) double-cuspidal forms, and hence is an eigenform of all Hecke operators. A computation using [6] and (2) yields that $\mathcal{T}_{\theta} f=\theta^{4} f$. (It should be noted however that this $f$ doesn't have an A-expansion in the sense of Petrov [5]). The conditions of Theorem 2.3 are satisfied with $l=1$ and $N_{1}=1$. As $a_{2}(f)=1$, our result implies that for all $i \geq 0$

$$
a_{1+3^{i}}(f)=A(\{i\} ; \theta)
$$

and by computing the first few coefficients of $f$ we see that $A(\{0\} ; \theta)=1, a_{4}=A(\{1\} ; \theta)=$ $\theta^{3}-\theta$, and $a_{10}=A(\{2\} ; \theta)=0$. In fact, applying formula (21) from the Section 5 below to this example yields that $a_{1+3^{i+1}}$ is a multiple of $a_{1+3^{i}}$, and it thus follows that $A(\{i\} ; \theta)=0$ for all $i \geq 2$. Furthermore, Theorem 2.3 also implies that $a_{1+3^{i}}(F)=a_{1+3^{i}}(f)$ for any type 0 double-cuspidal normalized form $F$ satisfying $\mathcal{T}_{\theta}(F)=\theta^{4} F$, regardless of its weight. Indeed, this can be verified directly for $F:=h^{2} g^{6}+\left(\theta^{3}-\theta\right) h^{4} g^{2} \in M_{20,0}^{2}$.

Another way to state our theorem is that if the power $N$ of the eigensystem is such that $N-1$ has exactly $l \leq q-1$ entries in its base $q$ expansion (counting multiplicities), then all the coefficients whose indices have the same property as $N$ are polynomial multiples of $a_{1+l}$. The use of multisets to handle the entries of the base $q$ expansion is thus natural as repetitions are commonplace. For example, the multiset corresponding to the base 5 expansion of $N-1=7$ is $\{0,0,1\}$, and thus the $l$ corresponding to $N=8$ is 3 . We hope that the (rather standard) usage of the same symbol for sets and multisets will not cause confusion, and we will strive to make the distinction clear whenever needed. (For instance, the symbol $v$ will always be associated with multisets.)

\section{Combinatorial properties of successive difference operators}

Define a sequence of difference operators acting on $\mathbb{F}_{q}(z)$ (where $z$ is any indeterminate) inductively as follows: $\mathcal{D}_{0}(f ; z):=f(z), \mathcal{D}_{1}(f ; z):=f(z+1)-f(z)$, and

$$
\mathcal{D}_{M+1}(f ; z):=\mathcal{D}_{M}(f ; z+1)-\mathcal{D}_{M}(f ; z)=\mathcal{D}_{1}\left(\mathcal{D}_{M}(f ; z) ; z\right)
$$


(We shall often drop the variable of the function when it is clear from the context). Note that $\mathcal{D}_{M}$ is linear in $f$, so to study its effect on polynomials or power series in $z$ it suffices to study its action on powers of $z$. Given a multiset $v=\left\{v_{1}, \ldots, v_{l}\right\}$ write

$$
q^{\nu}:=q^{\nu_{1}}+\cdots+q^{\nu_{l}} .
$$

We also declare $q^{\emptyset}:=0$.

If $I$ is any subset (in the usual sense) of $I(l):=\{1,2, \ldots, l\}$, then we let $v(I)$ denote the multiset

$$
v(I):=\left\{v_{i}: i \in I\right\} .
$$

Note that $v(I(l))=v$, whereas $v(\emptyset)=\emptyset$. Using this notation, it is straightforward to obtain the following formulas

$$
\begin{aligned}
& \mathcal{D}_{1}\left(z^{q^{v}}\right)=\sum_{I_{1} \subsetneq I(l)} z^{q^{v\left(I_{1}\right)}}, \\
& \mathcal{D}_{2}\left(z^{q^{v}}\right)=\sum_{I_{1} \subsetneq I(l)} \sum_{I_{2} \subsetneq I_{1}} z^{v^{\left(I_{2}\right)}}, \\
& \mathcal{D}_{3}\left(z^{q^{v}}\right)=\sum_{I_{1} \subsetneq I(l)} \sum_{I_{2} \subsetneq I_{1}} \sum_{I_{3} \subsetneq I_{2}} z^{q^{v\left(I_{3}\right)}},
\end{aligned}
$$

and a clear pattern emerges. We shall now introduce some notation and combinatorial results that will give more explicit formulas for the quantity $\mathcal{D}_{n}\left(z^{q^{v}}\right)$. Given sets (again in the usual sense) $A$ and $B$ and an integer $n \geq 1$, consider the set $\mathfrak{C}(A ; B ; n)$ of chains of $n$ strict set-inclusions between $B$ and $A$; namely

$$
\mathfrak{C}(A ; B ; n):=\left\{\left(I_{1}, I_{2}, \ldots, I_{n-1}\right): B \subsetneq I_{n-1} \subsetneq \cdots \subsetneq I_{1} \subsetneq A\right\} .
$$

Note that $\mathfrak{C}(A ; B ; n)=\emptyset$ unless $B \subsetneq A$ and their set difference satisfies $|A \backslash B| \geq n$. (If $B \subsetneq A$ and $|A \backslash B|=1$ we have $\mathfrak{C}(A ; B ; 1)=\{\emptyset\}$, corresponding to the only possible strict set inclusion, namely $B \subsetneq A$ ). As in (6) we have

$$
\begin{aligned}
\mathcal{D}_{n}\left(z^{q^{\nu}}\right) & =\sum_{I_{n} \subsetneq I(l)} \sum_{\mathfrak{c} \in \mathfrak{C}\left(I(l) ; I_{n} ; n\right)} z^{q^{\nu\left(I_{n}\right)}} \\
& =\sum_{e=n}^{l} \sum_{B \subsetneq I(l),|I(l) \backslash B|=e}|\mathfrak{C}(I(l) ; B ; n)| z^{q^{\nu(B)}} .
\end{aligned}
$$

\section{Example 3.1.}

$$
\begin{aligned}
& \mathcal{D}_{2}\left(z^{q^{\left\{\nu_{1}, v_{2}, v_{3}, v_{4}\right\}}}\right)=\sum_{e=2}^{4} \sum_{B \subsetneq I(4),|I(4) \backslash B|=e}|\mathfrak{C}(I(4) ; B ; 2)| z^{q^{\nu(B)}} \\
& =2\left(z^{q^{\nu_{1}}+q^{\nu_{2}}}+z^{q^{v_{1}}+q^{\nu_{3}}}+z^{q^{\nu_{1}}+q^{\nu_{4}}}+z^{q^{\nu_{2}}+q^{\nu_{3}}}+z^{q^{\nu_{2}}+q^{\nu_{4}}}+z^{q^{\nu_{3}}+q^{\nu_{4}}}\right) \\
& +6\left(z^{q^{v_{1}}}+z^{q^{\nu_{2}}}+z^{q^{\nu_{3}}}+z^{q^{v_{4}}}\right)+14 .
\end{aligned}
$$

Lemma 3.2. Consider the sequence of functions $f_{n}(x)$ defined by $f_{1}(x)=1$ and for $n \geq 2$

$$
f_{n}(x)=\sum_{i=0}^{n-1}(-1)^{i}\left(\begin{array}{c}
n \\
i
\end{array}\right)(n-i)^{x},
$$

then

1. For integers e the values $f_{n}(e)$ satisfy the recursion

$$
h_{n+1}(e)=\sum_{i=n}^{e-1}\left(\begin{array}{l}
e \\
i
\end{array}\right) h_{n}(i),
$$

with $h_{1}(e)=1$ for all e. Furthermore, (for $\left.n \geq 2\right) f_{n}(e)=0$ for $1 \leq e \leq n-1$. 
2. If $B \subsetneq A$ with $|A \backslash B|=e$, then

$$
|\mathfrak{C}(A ; B ; n)|=f_{n}(e) .
$$

3. $\quad$ For $n \geq 1, f_{n}(n)=n !$.

Proof. 1. The proof shall proceed by induction. Note that a solution to (9) with an initial condition is unique. By definition $f_{1}(e)=1=h_{1}(e)$, giving the induction base. For the induction step, assume $h_{n}(e)=f_{n}(e)$ for all $e$ and that $h_{n}(e)=0$ for $1 \leq e \leq n-1$ (this is satisfied trivially for $n=1$ ). The recurrence implies that $h_{n+1}(1)=\cdots=h_{n+1}(n)=0$. Also, the vanishing of those initial values enables us to start the sum in (9) with $i=1$. Using the induction hypothesis and binomial theorem we compute

$$
\begin{aligned}
h_{n+1}(e) & =\sum_{i=1}^{e-1}\left(\begin{array}{l}
e \\
i
\end{array}\right) \sum_{j=0}^{n-1}(-1)^{j}\left(\begin{array}{c}
n \\
j
\end{array}\right)(n-j)^{i} \\
& =\sum_{j=0}^{n-1}(-1)^{j}\left(\begin{array}{l}
n \\
j
\end{array}\right) \sum_{i=1}^{e-1}\left(\begin{array}{l}
e \\
i
\end{array}\right)(n-j)^{i} \\
& =\sum_{j=0}^{n-1}(-1)^{j}\left(\begin{array}{c}
n \\
j
\end{array}\right)\left((n+1-j)^{e}-(n-j)^{e}-1\right) \\
& =(n+1)+\sum_{j=1}^{n-1}(-1)^{j}(n+1-j)^{e}\left(\left(\begin{array}{l}
n \\
j
\end{array}\right)+\left(\begin{array}{c}
n \\
j-1
\end{array}\right)\right) \\
& +(-1)^{n}\left(\begin{array}{c}
n \\
n-1
\end{array}\right)+(-1)^{n} \\
& =\sum_{j=0}^{n}(-1)^{j}(n+1-j)^{e}\left(\begin{array}{c}
n+1 \\
j
\end{array}\right)=f_{n+1}(e),
\end{aligned}
$$

and the result follows.

2. We prove the formula by induction on $n$. When $n=1$ then for all $e \geq 1$,

$|\mathfrak{C}(A ; B ; 1)|=1=f_{1}(e)$ since $\mathfrak{C}(A ; B ; 1)=\{\emptyset\}$. Assume the statement true for $n$, and note that if $e \leq n$ then $\mathfrak{C}(A ; B ; n+1)=\emptyset$ and also $f_{n+1}(e)=0$ by part (1); which proves the formula in this case. Otherwise, if $e>n$ then for all $n \leq i \leq e-1$ there are $\left(\begin{array}{l}e \\ i\end{array}\right)$ choices for $I_{1}$ such that $B \subsetneq I_{1} \subsetneq A$ with $\left|I_{1} \backslash B\right|=i$. We also have

$$
\mathfrak{C}(A ; B ; n+1)=\bigcup_{I_{1} \subsetneq A}\left\{\left(I_{1}, I_{2}, \ldots, I_{n}\right):\left(I_{2}, \ldots, I_{n}\right) \in \mathfrak{C}\left(I_{1} ; B ; n\right)\right\} .
$$

Note that the union is clearly disjoint as the index $I_{1}$ 'colors' all of the entries corresponding to it. Thus the inductive hypothesis gives

$$
|\mathfrak{C}(A ; B ; n+1)|=\sum_{i=n}^{e-1}\left(\begin{array}{l}
e \\
i
\end{array}\right) f_{n}(i)=f_{n+1}(e),
$$

which proves the result.

3. By part (2), we can use any pair $B \subsetneq A$ with $|A \backslash B|=n$ to compute $f_{n}(n)$ as $|\mathfrak{C}(A ; B ; n)|$. The result now follows by realizing that the strict inclusion chains in $\mathfrak{C}(\{1,2, \ldots, n\} ; \emptyset ; n)$ correspond bijectively to permutations of the elements of $\{1,2, \ldots, n\}$. 
Example 3.3. The first few functions $f_{n}(x)$ are given by

$$
\begin{aligned}
& f_{1}(x)=1 \\
& f_{2}(x)=2^{x}-2, \\
& f_{3}(x)=3^{x}-3 \cdot 2^{x}+3 \\
& f_{4}(x)=4^{x}-4 \cdot 3^{x}+6 \cdot 2^{x}-4 .
\end{aligned}
$$

Corollary 3.4. Let $v=\left\{v_{1}, \ldots, v_{l}\right\}$ be a multiset of length $l \leq q-1$, then

1. $\mathcal{D}_{l}\left(z^{q^{v}}\right)=l$ !.

2. $\mathcal{D}_{M}\left(z^{q^{v}}\right) \neq 0$ for $0 \leq M \leq l$. and $\mathcal{D}_{M}\left(z^{q^{v}}\right)=0$ for $M \geq l+1$.

3. The operators $\mathcal{D}_{M}$ vanish identically for $M \geq q$.

Proof. Using (7) and Lemma 3.2 (and its notation) we see that

$$
\mathcal{D}_{n}\left(z^{q^{\nu}}\right)=\sum_{e=n}^{l} \sum_{B \subsetneq I(l),|I(l) \backslash B|=e} f_{n}(e) z^{q^{\nu(B)}} .
$$

In particular, for $l \leq q-1$ we have $\mathcal{D}_{l}\left(z^{q^{v}}\right)=f_{l}(z) z^{\nu(\emptyset)}=l ! \neq 0 \in \mathbb{F}_{q}$. It also follows that $\mathcal{D}_{M}\left(z^{q^{v}}\right)$ can't be zero for any $M<l$ as that would have forced it to be zero when $M=l$, whereas we clearly get $\mathcal{D}_{l+1}\left(z^{q^{v}}\right)=\mathcal{D}_{1}(l !)=0$. Part (3) follows easily from the vanishing of $\mathcal{D}_{q}$, which can be seen by noticing that $f_{q}(x) \equiv 0(\bmod q)$ as all the binomial coefficients in (8) would vanish for that index.

\section{Vanishing of certain binomial coefficients}

In [2], Gekeler obtained from (2) an explicit formula for the action of Hecke operators at degree one monic primes $\mathfrak{p}$ as follows

$$
\begin{aligned}
a_{n}\left(\mathcal{T}_{\mathfrak{p}} f\right)= & \mathfrak{p}^{k}\left(\sum_{j, s \geq 0, j q+s(q-1)=n}(-1)^{s}\left({ }^{j+s-1} s\right)^{s} a_{j}\right) \\
& +\sum_{i=0}^{n-1}\left(\begin{array}{c}
n-1 \\
i
\end{array}\right) \mathfrak{p}^{n-i} a_{n+i(q-1)}
\end{aligned}
$$

The main purpose of this section is to prove the vanishing of the first summand in the above expression for a doubly cuspidal form $f$ at the indices that we are studying. Since for such $f$ we have $a_{0}=a_{1}=0$, we may restrict our attention to $j \geq 2$ in that summand. This provides the motivation for the conditions in the following proposition, which nonetheless is ultimately a purely combinatorial statement.

Proposition 4.1. Let $v=\left\{v_{1}, \ldots, v_{l}\right\}$ be a multiset of nonnegative integers of length $l \leq q-1$. If $j \geq 2$ and $s \geq 0$ are integers for which

$$
j q+s(q-1)=1+q^{v},
$$

then

$$
\left(\begin{array}{c}
j+s-1 \\
s
\end{array}\right) \equiv 0 \quad(\bmod q)
$$

In preparation for the proof, we introduce some additional notation that will help streamline the discussion, and also make some useful remarks. First, for $v$ as in the statement of the proposition, we define integers $V_{i}:=V_{i}(v):=\left|\left\{v_{j}=i: 1 \leq j \leq l\right\}\right|$ for 
$0 \leq i \leq v_{l}$ (equivalently, $V_{i}$ is the $i^{\text {th }}$ digit of the base $q$ expansion of $q^{\nu}$ ). For convenience we shall also assume, without loss of generality, that $v_{i} \leq v_{j}$ for $i \leq j$. We thus have

$$
1+V_{0}+V_{1} q+\cdots+V_{\nu_{l}} q^{\nu_{l}}=1+q^{\nu_{1}}+\cdots+q^{\nu_{l}},
$$

and

$$
\sum_{i=0}^{v_{l}} V_{i}=l .
$$

Reducing (13) modulo $q$ and $q-1$, we see that any pair $(j, s)$ solving it must satisfy $s \equiv-1-V_{0} \quad(\bmod q)$ and $j \equiv 1+l \quad(\bmod q-1)$. Writing $X:=X(j, s):=j+s-1$, we can solve for either $s$ or $j$ in $j q+s(q-1)=1+q^{v}$ to obtain $X$ as function solely of $j$ or $s$. This function is increasing in $s$ and decreasing in $j$. Employing the above congruence for $s$, it is plain to see that the smallest value for $s \geq 0$ satisfying (13) is $s_{\min }=q-1-V_{0}$, and the corresponding value of $j$, which is the largest value of $j$ satisfying the same equation, is

$$
\begin{aligned}
j_{\max } & =\frac{1}{q}\left(1+V_{0}+V_{1} q+\cdots+V_{v_{l}} q^{\nu_{l}}-(q-1)\left(q-1-V_{0}\right)\right) \\
& =\left(2+V_{0}+V_{1}\right)+\left(V_{2}-1\right) q+\sum_{i=3}^{v_{l}} V_{i} q^{i-1} .
\end{aligned}
$$

It follows that the minimum value of $X$ is

$$
X_{\min }=j_{\max }+s_{\min }-1=V_{1}+V_{2} q+V_{3} q^{2}+\cdots+V_{v_{l}} q^{\nu_{l}-1} .
$$

As our focus is on $j \geq 2$, we are thus only interested in the cases $j_{\max } \geq 2$, which by (14) is satisfied if and only if $V_{i} \geq 1$ for some $i \geq 2$, or equivalently if $v_{l} \geq 2$ (remembering that $\left.V_{0}+V_{1} \leq \sum_{i=0}^{v_{l}} V_{i}=l \leq q-1\right)$. We shall thus make the assumption $v_{l} \geq 2$ for the remainder of this section. From that we get $j_{\min }=l+1$, and

$$
s_{\max }=q-1-V_{0}+\left(V_{2}+\cdots+V_{\nu_{l}}-1\right) q+\left(V_{3}+\cdots+V_{v_{l}}\right) q^{2}+\cdots+V_{\nu_{l}} q^{\nu_{l}-1} \geq 0 .
$$

Hence the maximum value of $X$ is

$$
X_{\max }=l-1-V_{0}+\left(V_{2}+\cdots+V_{\nu_{l}}\right) q+\left(V_{3}+\cdots+V_{\nu_{l}}\right) q^{2}+\cdots+V_{\nu_{l}} q^{\nu_{l}-1} .
$$

A simple calculation shows that any such $s$ (resp. $X$ ) between $s_{\min }$ and $s_{\max }$ (resp. $X_{\min }$ and $X_{\max }$ ) is of the form $s=s_{\min }+\alpha q$ (resp. $\left.X=X_{\min }+\alpha\right)$ for some $0 \leq \alpha \leq \alpha_{\max }$, with $\alpha_{\max }$ given by

$$
\begin{aligned}
\alpha_{\max } & :=X_{\max }-X_{\min }=\frac{s_{\max }-s_{\min }}{q} \\
& =\left(V_{2}+\cdots+V_{v_{l}}-1\right)+\left(V_{3}+\cdots+V_{v_{l}}\right) q+\cdots+V_{v_{l}} q^{\nu_{l}-2} .
\end{aligned}
$$

By our assumptions $v_{l} \geq 2$ and $l \leq q-1$, we see that (16) gives the base $q$ expansion of $\alpha_{\max }$. In addition, if $0 \leq \alpha \leq \alpha_{\max }$ has base $q$ expansion

$$
\alpha=\alpha_{0}+\alpha_{1} q+\cdots+\alpha_{\nu_{l}-2} q^{\nu_{l}-2},
$$

and if $0 \leq j \leq v_{l}-3$ is such that $\alpha_{i}=\left(\alpha_{\max }\right)_{i}$ for $j+1 \leq i \leq v_{l}-2$ (where we use $(n)_{i}$ to denote the $i^{\text {th }}$ digit in the base $q$ expansion of $n$ ), then the maximality of $\alpha_{\max }$ implies $\alpha_{j} \leq\left(\alpha_{\max }\right)_{j}$.

Proof. By the above discussion, we need to show that for all $0 \leq \alpha \leq \alpha_{\max },\left(\begin{array}{l}X_{\min }+\alpha \\ s_{\min }+q \alpha\end{array}\right) \equiv$ $0(\bmod q)$. Recall that Lucas' Theorem states that if $0 \leq \beta_{i}, \delta_{i} \leq q-1$ then

$$
\left(\begin{array}{c}
\sum_{i=0}^{n} \beta_{i} q^{i} \\
\sum_{i=0}^{n} \delta_{i} q^{i}
\end{array}\right) \equiv \prod_{i=0}^{n}\left(\begin{array}{c}
\beta_{i} \\
\delta_{i}
\end{array}\right) \quad(\bmod q),
$$


with the customary convention $\left(\begin{array}{l}n \\ j\end{array}\right)=0$ whenever $n<j$. Thus our assertion will follow if we show that $\left(X_{\min }+\alpha\right)_{i}<\left(s_{\min }+q \alpha\right)_{i}$ for at least one value of $i$. Note that $\left(s_{\min }+q \alpha\right)_{0}=$ $q-1-V_{0}$ and for $1 \leq i \leq v_{l}-1,\left(s_{\min }+q \alpha\right)_{i}=\alpha_{i-1}$. If $0 \leq \alpha_{0}<q-1-V_{0}-V_{1}$ then $\left(X_{\min }+\alpha\right)_{0}=\alpha_{0}+V_{1}<q-1-V_{0}$. Also, if $q-V_{1} \leq \alpha_{0} \leq q-1$, then $\left(X_{\min }+\alpha\right)_{0}=$ $\alpha_{0}+V_{1}-q \leq V_{1}-1<q-1-V_{0}$. Thus $\left(X_{\min }+\alpha\right)_{0}<\left(s_{\min }+q \alpha\right)_{0}$ unless

$$
q-1-V_{0}-V_{1} \leq \alpha_{0} \leq q-1-V_{1} .
$$

By a similar argument, we get that

$$
\left(X_{\min }+\alpha\right)_{1}<\left(s_{\min }+q \alpha\right)_{1}=\alpha_{0} \text { unless } \alpha_{0}-V_{2} \leq \alpha_{1} \leq q-1-V_{2} .
$$

Assume (17) and let $j$ be an index in the range $1 \leq j \leq v_{l}-3$ for which the inequalities

$$
\alpha_{i-1}-V_{i+1} \leq \alpha_{i} \leq q-1-V_{i+1}
$$

are satisfied for $1 \leq i \leq j$ but not for $i=j+1$. Under this condition we deduce that $\left(X_{\min }+\alpha\right)_{j+1}<\left(s_{\min }+q \alpha\right)_{j+1}=\alpha_{j}$. To see this, note that (15), (17) and (18) imply that $\left(X_{\min }+\alpha\right)_{i}=\left(X_{\min }\right)_{i}+\alpha_{i}$ for $0 \leq i \leq j$ (i.e. no "carries" between any of the first $j$ digits and the next one in the base $q$ expansion of the sum). For $i=j+1$ we either have $0 \leq \alpha_{j+1}<\alpha_{j}-V_{j+2}$, from which it easily follows that $\left(X_{\min }+\alpha\right)_{j+1}=\alpha_{j+1}+V_{j+2}<\alpha_{j}$, or else $q-V_{j+2} \leq \alpha_{j+1} \leq q-1$, and hence $\left(X_{\min }+\alpha\right)_{j+1}=\alpha_{j+1}+V_{j+2}-q \leq V_{j+2}-1<\alpha_{j}$. The last inequality is equivalent to $1+\alpha_{j}-V_{j+2}>0$, which follows from noticing that the lower bounds in (17) and (18) give $q-1-V_{0}-V_{1}-\cdots-V_{i+1} \leq \alpha_{i}$ for $0 \leq i \leq j \leq v_{l}-3$ and hence

$$
1+\alpha_{i}-V_{i+2} \geq q-V_{0}-V_{1}-\cdots-V_{i+1}-V_{i+2} \geq q-l \geq 1 .
$$

Thus, it only remains to show that assuming (17), we cannot have (18) for all $1 \leq i \leq v_{l}-2$. Indeed, if we did then for all $0 \leq i \leq v_{l}-2$

$$
\alpha_{i} \geq q-1-V_{0}-V_{1}-\cdots-V_{i+1}=(q-1)-l+V_{i+2}+V_{i+3}+\cdots+V_{\nu_{l}-1}+V_{v_{l}} .
$$

In particular

$$
\alpha_{v_{l}-2} \geq(q-1)-l+V_{v_{l}}
$$

but $\left(\alpha_{\max }\right)_{v_{l}-2}=V_{v_{l}}$, so we must thus have $l=(q-1)$ and $\alpha_{v_{l}-2}=V_{v_{l}}$. substituting those values in (19) then gives $\alpha_{i}=\left(\alpha_{\max }\right)_{i}$ for $1 \leq i \leq v_{l}-2$ and $\alpha_{0}>\left(\alpha_{\max }\right)_{0}=$ $V_{2}+V_{3}+\cdots+V_{v_{l}}-1$, a contradiction.

\section{Proof of Theorem 2.3}

First, we introduce a couple more conventions and abbreviations to lighten the notation of the formulas. Given a multiset $v=\left\{v_{1}, \ldots, v_{l}\right\}$ and a set $I \subset\{1,2, \ldots, l\}$, we write $\widehat{v}(I):=\left\{v_{j}: j \notin I\right\}$ and

$$
v^{+}(I):=\left\{1+v_{i}: i \in I\right\} \cup \widehat{v}(I) .
$$

(Both $\widehat{v}(I)$ and $v^{+}(I)$ are multisets in general). Note that $v^{+}(\emptyset)=\widehat{v}(\emptyset)=v$, and that $|\widehat{v}(I)|=l-|I|$ whereas $\left|v^{+}(I)\right|=l$ always. 
Lemma 5.1. For $f=\sum a_{i} u^{i} \in M_{k, m}^{2}$ and $v=\left\{v_{1}, \ldots, v_{l}\right\}$ a multiset of nonnegative integers of length $l \leq q-1$ we have

$$
\begin{aligned}
a_{1+q^{v}}\left(\mathcal{T}_{\theta} f\right) & =\sum_{i=0}^{q^{v}}\left(\begin{array}{c}
q^{v} \\
i
\end{array}\right) \theta^{1+q^{v}-i} a_{1+q^{v}+i(q-1)} \\
& =\sum_{I \subset\{1, \ldots, l\}}\left(\begin{array}{c}
q^{v} \\
q^{v(I)}
\end{array}\right) \theta^{1+q^{v(I)}} a_{1+q^{v^{+}(I)}}
\end{aligned}
$$

Proof. Applying Proposition 4.1 to (12) results in the first equality in (20), and the second one follows by noticing that, by using Lucas' Theorem as in the proof of Proposition 4.1, $\left(\begin{array}{c}q^{v} \\ i\end{array}\right) \equiv 0(\bmod q)$ whenever $i$ is not of the form $q^{v(I)}$ for some $I \subset I(l)$.

Proof. If $f \in M_{k, m}^{2}$ satisfies $\mathcal{T}_{\theta}(f)=\theta^{N} f$ with $N=1+q^{N_{1}}+\cdots+q^{N_{l}}$ then (20) gives

$$
\theta^{q^{N_{1}}+q^{N_{2}}+q^{N_{3}}+\cdots+q^{N_{l}}} a_{1+q^{v}}=\sum_{I \subset\{1, \ldots, l\}}\left(\begin{array}{c}
q^{v} \\
q^{v(I)}
\end{array}\right) \theta^{q^{\widehat{v}(I)}} a_{1+q^{\nu+(I)}} .
$$

Note that the index of the coefficients in (4) is completely invariant under permutations of elements of $v$, so without loss of generality we shall assume throughout the proof that

$$
0 \leq v_{1} \leq \cdots \leq v_{l}
$$

The proof will utilize induction on $v_{l}=\max v$. The base case $v_{l}=0$ is immediate, and we can take $A(\{0,0, \ldots, 0\} ; \theta)=1$. For the induction step, assume the theorem is true (i.e. $a_{1+q^{v}}=A(v ; \theta) a_{1+l}$ with $\left.A(v ; \theta) \in \mathbb{F}_{q}[\theta]\right)$ for all $v$ with $v_{l}=n$, and let

$$
C(n ; v):=\left|\left\{i: 0 \leq i \leq l, v_{i}=n\right\}\right| .
$$

(So being true for $v_{l}=n$ implies the theorem is true for all $v$ such that $1 \leq C(n ; v) \leq l$.) We proceed to show that the statement is true for all $\mu=\left\{\mu_{1}, \ldots, \mu_{l}\right\}$ with $\mu_{l}=n+1$ by (another) induction on $C(n+1 ; \mu)$. If $C(n+1 ; \mu)=1$ then $\mu=v^{+}(\{l\})$ where $v$ is defined by $v_{l}=n$ and $v_{i}=\mu_{i} \leq n$ for all $1 \leq i \leq l-1$. Using Corollary 3.4 as well as Lemma 3.2 and its notation, we see that $\mathcal{D}_{l-1}$ will annihilate $\theta^{q^{\widehat{V}(I)}}$ if and only if $|I| \geq 2$. We also get

$$
\begin{aligned}
& \mathcal{D}_{l-1}\left(\theta^{q^{\widehat{v}(())}}\right)=\mathcal{D}_{l-1}\left(\theta^{q^{v}}\right)=(l-1) !\left(\theta^{q^{\nu_{1}}}+\cdots+\theta^{q^{\nu_{l}}}\right)+f_{l-1}(l), \\
& \mathcal{D}_{l-1}\left(\theta^{q^{\widehat{v}(i i))}}\right)=(l-1) !,
\end{aligned}
$$

and $\left(\begin{array}{c}q^{v} \\ q^{v_{i}}\end{array}\right)=V_{v_{i}}$. Let $E(v):=\left\{1 \leq i \leq l: v_{i}=v_{l}\right\}$. Obviously $l \in E(v)$ and we have (again with the above choice of $v)|E(v)|=V_{n}$ and $\mu=v^{+}(\{i\})$ for all $i \in E(v)$. Hence, applying $\mathcal{D}_{l-1}$ to (21) with that $v$ gives

$$
V_{n}^{2} a_{1+q^{\mu}}=\sum_{i=1}^{l}\left(\theta^{q^{N_{i}}}-\theta^{q^{v_{i}}}\right) a_{1+q^{\nu}}-\sum_{i \notin E(v)} V_{v_{i}} a_{1+q^{\left.\nu^{+}(i i)\right)} .}
$$

All of the multisets on the right hand side (namely $v$ and $v^{+}(\{i\})$ for $i \notin E(v)$ ) have maximal entry $v_{l}=n$, and thus each of the coefficients on the right hand side is a polynomial (in $\theta$ ) multiple of $a_{1+l}$ by the induction hypothesis; thus establishing the base case for the induction on $C(n+1 ; \mu)$. 
Next we consider $C(n+1 ; \mu)=e$. Write $\mu=v^{+}(\{l-e+1, l-e+2, \ldots, l\})$ with $v=\left\{\mu_{1}, \ldots, \mu_{l-e}, n, n, \ldots, n\right\}\left(0 \leq v_{i}=\mu_{i} \leq n\right.$ for $\left.1 \leq i \leq l-e\right)$. Note that $v^{+}(I)=\mu$ if and only $I \subset E(v)$ and $|I|=e$. Applying $\mathcal{D}_{l-e}$ to this $v$ and using (21) we get

$$
\begin{aligned}
& \mathcal{D}_{l-e}\left(\theta^{\left.q^{\left\{N_{1}, \ldots, N_{l}\right\}}-\theta^{q^{\nu}}\right) a_{1+q^{v}}}\right. \\
& =\sum_{\emptyset \neq I \subset\{1, \ldots, l\},|I| \leq e, \nu^{+}(I) \neq \mu}\left(\begin{array}{c}
q^{v} \\
q^{v(I)}
\end{array}\right) \mathcal{D}_{l-e}\left(\theta^{q^{\widehat{q}(I)}}\right) a_{1+q^{\nu^{+}(I)}} \\
& +\sum_{I \subset E(v),|I|=e}\left(\begin{array}{c}
q^{v}(I) \\
q^{(I)}
\end{array}\right) \mathcal{D}_{l-e}\left(\theta^{q^{\widehat{v}(I)}}\right) a_{1+q^{\mu} .}
\end{aligned}
$$

The number of subsets $I \subset E(v)$ with $|I|=e$ is clearly $\left(\begin{array}{c}V_{n} \\ e\end{array}\right)$, and for each such $I$ we also have $\left(\begin{array}{c}q^{v} \\ q^{v(I)}\end{array}\right) \equiv\left(\begin{array}{c}V_{n} \\ e\end{array}\right)(\bmod q)$ by Lucas' Theorem. Hence

$$
\begin{aligned}
& (l-e) !\left(\begin{array}{c}
V_{n} \\
e
\end{array}\right)^{2} \cdot a_{1+q^{\mu}}=\mathcal{D}_{l-e}\left(\theta^{\left.q^{\left\{N_{1}, \ldots, N_{l}\right\}}-\theta^{q^{\nu}}\right) a_{1+q^{\nu}}}\right. \\
& -\sum_{\emptyset \neq I \subset\{1, \ldots, l\},|I| \leq e, \nu^{+}(I) \neq \mu}\left(\begin{array}{c}
q^{v} q^{\nu}(I) \\
)
\end{array} \mathcal{D}_{l-e}\left(\theta^{q^{\widehat{v}(I)}}\right) a_{1+q^{\nu^{+}(I)} .}\right.
\end{aligned}
$$

All of the multisets $v^{+}(I)$ on the right hand side either have $v_{l}=n$ (whenever $I \cap E(v)=$ $\emptyset)$, or else have $C\left(n+1 ; v^{+}(I)\right) \leq e-1$ (for $|I| \leq e$ we have $C\left(n+1, v^{+}(I)\right)=e$ if and only if $\left.v^{+}(I)=\mu\right)$. Thus the expression on the right hand side is a polynomial in $\theta$ by the inductions on $C(n+1 ; \mu)$ and on $n$, which establishes (4) for any length $l$ multiset $v$ and with $A(\nu ; \theta)$ polynomial. Lastly, note that we could use any $\tilde{\theta}=\theta+c$ with $c \in \mathbb{F}_{q}$ as a generator of $A$ over $\mathbb{F}_{q}$, and the corresponding parameter $\tilde{u}$ at the cusp will be equal to $u$. It also follows that if $\mathcal{T}_{\theta} f=\theta^{N} f$ then $\mathcal{T}_{\tilde{\theta}} f=\tilde{\theta}^{N} f$, and hence the coefficients of $f$ must be invariant under $\theta \mapsto \tilde{\theta}$, which yields $A(\nu ; \theta) \in \mathbb{F}_{q}\left[\theta^{q}-\theta\right]$ and completes the proof.

\section{Author details}

${ }^{1}$ Department of Mathematics, Faculty of Science Cairo University, Giza 12613, Egypt. ${ }^{2}$ Present Address: Texas A\&M University at Qatar, Science Program, Doha 23874, Qatar. ${ }^{3}$ Max Planck Institute for Mathematics, Vivatgasse 7, 53111 Bonn, Germany.

Received: 5 October 2015 Accepted: 27 December 2015

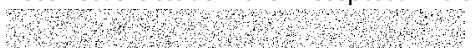

\section{References}

1. Armana, C: Coefficients of Drinfeld modular forms and Hecke operators. J. Number Theory. 131(8), 1435-1460 (2011)

2. Gekeler, E-U: On the coefficients of Drinfeld modular forms. Invent. Math. 93, 667-700 (1988)

3. Goss, D: Modular forms for $\mathbb{F}_{r}[t]$. J. Reine Angew. Math. 317, 16-39 (1980)

4. Goss, D: $\pi$-adic Eisenstein series for function fields. Compositio Math. 41(1), 3-38 (1980)

5. Petrov, A: A-expansions of Drinfeld modular forms. J. Number Theory. 133(7), 2247-2266 (2013). doi:10.1016/j.jnt.2012.12.012

6. Sage Mathematics Software (Version 4.8), The Sage Developers, (2014). http://www.sagemath.org 